\title{
Zone-Plate Based Nanospectroscopy with Soft X-Rays at the SLS
}

\author{
R.H. Fink*, C. Hub and G. Tzvetkov \\ Department Chemie und Pharmazie \\ and Interdisciplinary Center for Molecular Materials (ICMM) \\ Universität Erlangen-Nürnberg, Egerlandstr. 3, D-91058 Erlangen, Germany
}

\begin{abstract}
With the advance of nanostructuring techniques and the availability of third-generation synchrotron sources, Fresnel-type zone plate optics is widely used for nanospectroscopic investigations with resolutions of several $10 \mathrm{~nm}$. Whereas hard X-rays commonly use diffraction or elemental contrast (e.g. using X-ray fluorescence), soft X-rays are favored to utilize the spectroscopic contrast in the near-edge region of the absorption edge. In particular, for soft materials, the relevant $\mathrm{C}-, \mathrm{N}$ - and $\mathrm{O} 1 s$ absorption edges show distinct resonances which allow fingerprint-type chemical identification, even in water surroundings. Few selected examples from modern material science will be discussed to demonstrate that the combination of X-ray absorption spectroscopy with high spatial resolution yields new insight into the nanoscale properties of these materials.
\end{abstract}

PACS numbers: 68.37.Yz , 82.80.--d, 82.80.Ej, 07.85.Tt

\section{Introduction}

The ongoing miniaturization in science and technology demands for complementary techniques to investigate ultrasmall materials. Although several techniques exist which allow structural investigations down to the atomic level (e.g., transmission electron microscopy (TEM) or scanning probe techniques), the combination of microscopic and spectroscopic information is required to obtain information on structure-property relationships since many physical or chemical properties change for decreasing sizes. It seems to be a general rule that techniques which allow highest spatial resolution lack spectroscopic or chemical sensitivity and vice versa. X-ray induced electron spectroscopy techniques have developed into highly versatile analytical methods to explore the electronic structure of matter, either in high-resolution photoelectron spectroscopy (XPS) or in near-edge absorption fine structure (NEXAFS) [1]. Both spectroscopies can be combined with high spatial resolution: in case of photoemission, most commonly an electron microscope can be used to generate an energy-filtered 2D image (XPEEM) [2, 3], whereas in X-ray absorption focussed $\mathrm{X}$-rays are used and 2D image formation is achieved by raster-scanning the sample across the focal spot. Usually the transmitted intensity is recorded, therefore the technique is described as scanning-transmission X-ray microspectroscopy (STXM) [4, 5].

At present, several soft X-ray STXM instruments are

* corresponding author; e-mail: fink@chemie.uni-erlangen.de operated at the Advanced Light Source (ALS, Berkeley, USA) [6], NSLS (Brookhaven, USA) [7], CLS (Saskatoon, Canada) [8], BESSY (Berlin, Germany) [9], and ELETTRA (Trieste, Italy) [10] synchrotron radiation facilities. We have started with the operation of the PolLux-STXM at the Swiss Light Source (Paul Scherrer Institut, Villigen, Switzerland), which is an improved version of the ALS polymer STXM [11]. This paper summarizes the essential aspects of soft X-ray STXM. Few examples will be discussed from various fields of material science. From the spectroscopic information gained on the nanoscale new insights into the material properties are reported.

\section{Soft X-ray scanning transmission microspectroscopy}

The general principle of an STXM is shown in Fig. 1. The Fresnel zone plate (FZP) focuses the soft X-ray light to a diffraction limited spot. Higher diffraction orders are filtered out using an order-sorting aperture (OSA, diameter $70 \mu \mathrm{m}$ ) located between the FZP and the sample, which has to be sufficiently thin to prevent absorption saturation (see Sect. 3). The intensity of the transmitted X-ray beam is measured by either a photodiode or a photomultiplier tube coupled to a scintillator, which converts the X-rays to visible light. The photon energy dependent focal length of a zone plate $f(E)$ is given by $f(E)=d_{\mathrm{FZP}} \Delta r \frac{1}{c h} E$. For typical zone plate parameters (outer diameter $d_{\mathrm{FZP}}=240 \mu \mathrm{m}$, thinnest ring $\Delta r=35 \mathrm{~nm}$ ) this corresponds to an energy dependent focal length of $f(E)=a_{1} E=(7 \mu \mathrm{m} / \mathrm{eV}) E$. Imaging of samples mounted on the piezo-driven nanostage 
is accomplished by raster scanning across the X-ray focus. To achieve the high spatial resolution, closed loop optical interferometry is used to ensure the sample positioning with reproducibility in the range of few nanometers (including possible vibrational effects). Lateral resolutions about $20 \mathrm{~nm}$ were recently demonstrated for newest-generation zone plates [12].

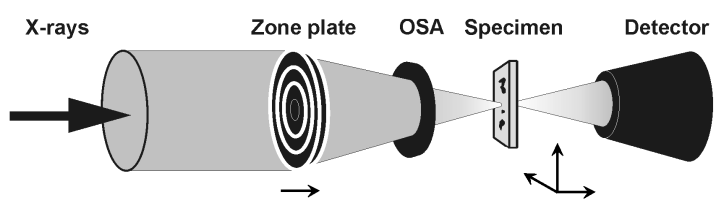

Fig. 1. Scheme of the scanning transmission X-ray microspectroscope (STXM). The Fresnel microzone plate focuses the X-rays onto the sample, which is raster scanned across the focussed beam. The OSA reduces the intensity from higher diffraction orders. The transmitted intensity is detected with a photomultiplier tube or Si diode.

\section{Sample requirements}

The basic contrast mechanism in STXM is based on the absorption of X-rays which are transmitted through the sample. The transmitted $I_{\text {trans }}$ is given by the LambertBeer expression

$$
I_{\text {trans }}(\boldsymbol{r})=I_{0} \exp (-\mu(\boldsymbol{P}, E, Z) t(\boldsymbol{r})),
$$

where $I_{0}$ and $I_{\text {trans }}(\boldsymbol{r})$ are the incident and transmitted $\mathrm{X}$-ray intensities at position $\boldsymbol{r}$, respectively. The absorption coefficient $\mu$ depends on the polarization $\boldsymbol{P}$, the photon energy $E$, and the atomic number $Z$. The local thickness of the sample is $t(\boldsymbol{r})$. The sensitivity to the chemical elements primarily comes from the dependence of the absorption edges, but variations in the chemical state lead to significant changes in the position and intensity of absorption peaks in the near-edge region thus providing detailed chemical information [13].

The samples can be either prepared on commercially available $\mathrm{Si}_{3} \mathrm{~N}_{4}$ membranes (thickness $\leq 100 \mathrm{~nm}$ ) or using transmission electron microscope grids. Typical sample thicknesses are in the range between 30 and $150 \mathrm{~nm}$. For biological samples, water environment with an overall thickness of up to $8 \mu \mathrm{m}$ (in between two thin $\mathrm{Si}_{3} \mathrm{~N}_{4}$ membranes) can be easily utilized. This particular aspect is possible due to the relatively low absorption of water for photon energies below the $\mathrm{O} 1 s$ absorption edge of water $(h \nu=535 \mathrm{eV})$. Thus, no staining is required as, e.g., in TEM since the contrast is mainly governed by the spectroscopic differences (see Sect. 4.3).

Since in general, synchrotron radiation is linearly polarized, the linear dichroism can be utilized to investigate orientational differences (e.g. in crystalline samples of organic materials, see Sect. 4.1). For anti/ferromagnetic samples the linear/circular magnetic dichroism can be employed. Prototype experiments to investigate the magnetization dynamics of ferromagnetic nanostructures were reported recently [14].

\section{Prototype STXM experiments}

In the following, we will present few prototype experiments of soft matter samples with particular emphasis on the combination of both, imaging and spectroscopic analysis. The examples were carefully chosen to underline the above mentioned aspects of STXM with respect to the various contrast mechanism due to element and chemical contrast, or sample thickness and dichroism.

\subsection{Orientational inhomogeneities in thin film organic field-effect transistors}

Organic-based devices like organic light-emitting diodes/devices (OLEDs) or organic field-effect transistors (OFETs) have recently attracted much attention due to cheaper production as thin-film devices or tailored organic substances [15]. However, epitaxial growth of organic films is more difficult than for inorganic semiconductors. Therefore, morphological inhomogeneities have negative impact on the electric transport properties in these devices.
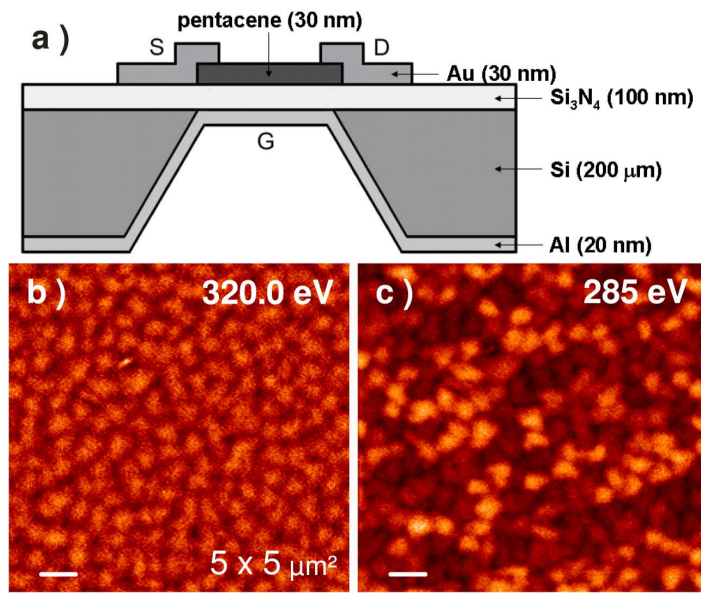

Fig. 2. (a) Schematic setup of an organic field-effect transistor with $\mathrm{Si}_{3} \mathrm{~N}_{4}$-dielectric. (b) and (c) STXM images from an ultrathin pentacene film used as active semiconductor layer in a field-effect transistor. The two chosen photon energies correspond to off-resonance (b) and on-resonance conditions (c), respectively (scanned area: $\left.5 \times 5 \mu \mathrm{m}^{2}\right)$.

We have successfully prepared pentacene-base field-effect transistors on ultrathin $\mathrm{Si}_{3} \mathrm{~N}_{4}$-membranes (100 nm thin), which serve as dielectric between the gate electrode and the organic semiconductor (pentacene thickness: $30 \mathrm{~nm}$; device scheme shown in Fig. 2a). Thus, the overall sample thickness is sufficiently small to investigate the OFET in situ in the STXM while it is operated. Figure $2 \mathrm{~b}$ shows an STXM image of the pentacene film in between the source and drain electrode. The photon energy was $320 \mathrm{eV}$, i.e. well above the $\mathrm{C} 1 s$ absorption edge 
of pentacene. Thus, all intensity variations are purely due to thickness differences. The non-uniformity reflects the polycrystalline nature of the pentacene film. Pentacene films on inert substrates are usually considered to consist of a bulk-like phase with almost upright standing molecules. Our present investigations differ from this assumption, as demonstrated in Fig. 2c. The image in Fig. 2c was recorded at a photon energy of $285 \mathrm{eV}$, which corresponds to the most intense resonance of pentacene. The intensity variations in Fig. 2c are no longer purely due to thickness variations but rather due to orientational inhomogeneities of the individual grains within the pentacene film. However, it is not straightforward possible to deduce the exact orientation of the individual domains since we expect herringbone-like molecular arrangement within the crystalline unit cell. In addition, the domain orientation with respect to the $E$-vector cannot unambiguously be determined. Further investigations of the pentacene OFETs during operation are presently carried out to monitor the local electronic changes during charge transport.

\subsection{Phase-change microcapsules}

Core-shell microstructures are considered in many technological areas, for e.g. in drug delivery or as nanocontainers. Here we show results from a STXM study of Micronal $^{\circledR}$ phase-change microcapsules which are microscopically small acrylic polymer shell capsules containing paraffin wax droplets in their cores [16]. Micro-encapsulated paraffin wax is a multihomologous mixture of $n$-alkanes $\left(\mathrm{C}_{n} \mathrm{H}_{2 n+2}\right)$ and its melting temperature is in the range between $23^{\circ} \mathrm{C}$ and $26^{\circ} \mathrm{C}$. For specimen preparation, one droplet of the microcapsules water suspension was deposited onto a $100 \mathrm{~nm}$ thick $\mathrm{Si}_{3} \mathrm{~N}_{4}$ membrane. Figure 3 shows STXM images which were recorded at $280 \mathrm{eV}$ (i.e. below the $\mathrm{C} 1 s$ absorption threshold to avoid absorption saturation) at various tempera-

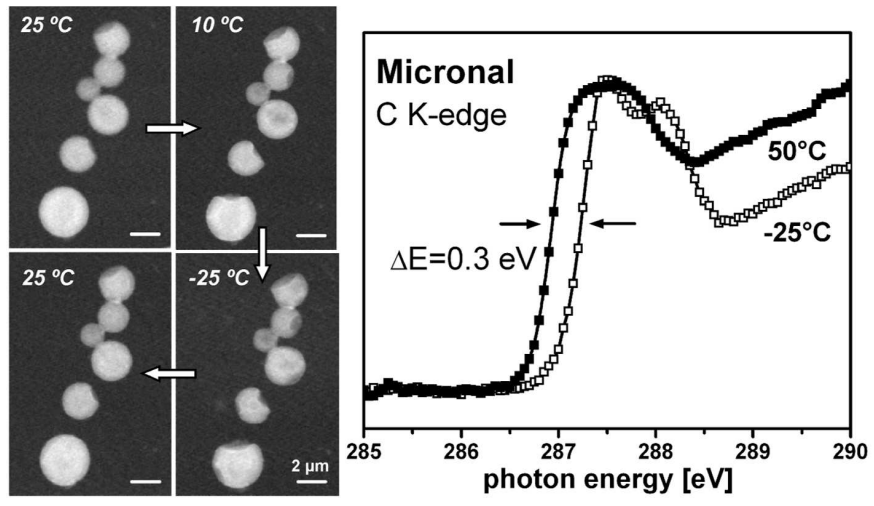

Fig. 3. STXM images from Micronal ${ }^{\circledR}$ microcapsules recorded at various temperatures showing reversible morphology changes. The NEXAFS spectra on the right hand side show the spectral changes in the quasi-crystalline and molten state of the interior paraffin material. tures below and above the phase transition temperature. At $25^{\circ} \mathrm{C}$ (Fig. 3) mostly spherical particles are detected, whereas for lower temperatures $\left(T=-25^{\circ} \mathrm{C}\right.$, Fig. 3$)$, the spherical shape of the microcapsules is lost due to contraction of the wax material inside the shell. Let us note that the STXM images recorded after warming the sample back up to room temperature $\left(25^{\circ} \mathrm{C}\right)$ is essentially the same as the first image taken at room temperature before cooling the microcapsules. The phase transition is furthermore detected spectroscopically in the $\mathrm{C} 1 \mathrm{~s}$ NEXAFS spectra of the wax material inside the Micronal $^{\circledR}$ capsules (Fig. 3) [17]. In the quasi-crystalline form of alkanes the spectrum is governed by a double resonance $(h \nu=287.5$ and $288.0 \mathrm{eV})$, which are associated with $\mathrm{C} 1 s \rightarrow \sigma_{\mathrm{C}-\mathrm{H}}^{*} /$ Rydberg transitions [18]. This double-peak structure is also detected in the Micronal ${ }^{\circledR}$ capsules below the phase transition temperature and we may therefore conclude that the alkane mixture inside the microspheres has crystalline character. Upon heating above the phase transition temperature, the double peaks transfer into a broadened single resonance which is red shifted. The spectral shifts are in accordance with theoretical and experimental studies on various alkanes in gas phase or in the condensed state. Weiss et al. performed detailed $a b$ initio calculations of isolated alkanes and those in a matrix of other alkanes [19], whereas Urquhart et al. investigated the NEXAFS spectra of neopentane experimentally [20]. The authors explained the spectral shift with a difference in the degree of Rydberg-valence mixing in the $\mathrm{C} 1 s \rightarrow \sigma_{\mathrm{C}-\mathrm{H}}^{*}$ transition with condensation in alkanes [20].

\subsection{Gas-filled microballoons}

Gas-encapsulated microballoons are of interest as contrast enhancers for ultrasound diagnostics and/or as carriers of therapeutic gases. Very recently, the PolLux-STXM has been utilized for the first spectromicroscopy examination of gas-filled microballoons in aqueous solution [21]. In the present example, stable air-filled poly(vinyl alcohol) (PVA)-coated microballoons were prepared by cross-linking telechelic PVA at the water/air interface. Figure 4 shows transmission images $\left(20 \times 20 \mu \mathrm{m}^{2}\right)$ of microballoons in a water environment recorded at (a) $520 \mathrm{eV}$ and (b) $550 \mathrm{eV}$. The absorption contrast in Fig. 4a, below the oxygen $K$-edge $(520 \mathrm{eV})$, is due to the strong absorption of the X-rays by the carbonaceous material, whereas the water and air have only weak absorption at this photon energy [21]. Above the oxygen $K$-edge $(550 \mathrm{eV})$, the transmission changes drastically. The inside of the microballoons stays transparent (white), while the surrounding water turns absorbing (dark). This is due to the high oxygen $1 s$-absorption in water and the low absorption in air primarily due to their density differences. The image shows some variations between the particles, i.e. differences in the polymeric shell thickness and different absorption from the internal part of the microballoons. Moreover, the microballoon labelled $B$ shows almost the same absorption signal as 


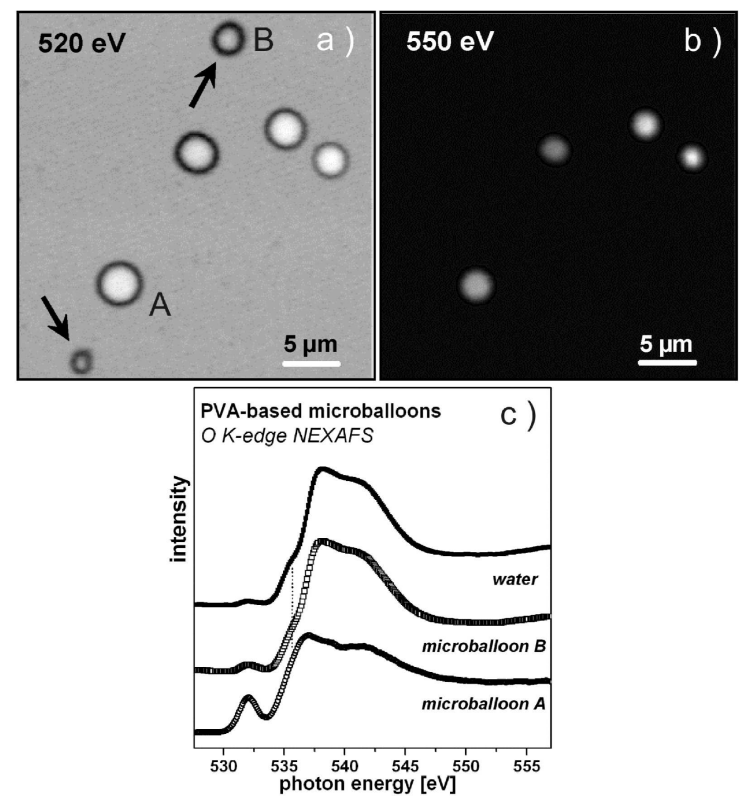

Fig. 4. STXM transmission images of microballoons in water environment recorded at a photon energy of $520 \mathrm{eV}$ (a) and $550 \mathrm{eV}$ (b). The objects marked with arrows are water-filled. (c) Oxygen $K$-edge NEXAFS spectra of the surrounding water and the interior parts of microballoons $A$ and $B$.

the surrounding water at $550 \mathrm{eV}$ (see Fig. 4b). This suggests that microballoon $B$ is filled with water which is corroborated by the oxygen $K$-edge NEXAFS spectra. The absorption spectra extracted from line scans across the inner part of the particles $A$ and $B$ are compared in Fig. 4c. A NEXAFS spectrum acquired from the surrounding water is also shown in this figure. The spectrum from microballoon $B$ shows essentially the same resonances as the water spectrum except a small intensity decrease in the feature at $535.4 \mathrm{eV}$ and an intensity increase in the peak at $532.1 \mathrm{eV}$. This result explicitly suggests the presence of water inside the balloon. In contrast to the spectra of the surrounding water and to particle $B$, the NEXAFS spectrum of the microballoon $A$ shows a strong resonance at $532.1 \mathrm{eV}$, which is assigned to the $\mathrm{O} 1 s \rightarrow \pi_{(\mathrm{C}=\mathrm{O})}^{*}$ transition originating from the carbonyl groups of the telechelic PVA shell. Hence, the NEXAFS spectrum of the microballoons $A$ shows only the resonances typical of the telechelic PVA shell. This result strongly suggests that microballoon $A$ is air-filled [21]. More recent investigations allowed to study the profiles of the stabilizing shell in more detail [22].

\subsection{Human hair}

Biofibres like e.g. spider webs or hair are extremely interesting materials due to their high ductile strength [23]. We have started to investigate human hair with respect to its interior morphology in connection with the spectral properties. Figure 5 shows a $20 \times 20 \mu \mathrm{m}^{2}$ area (average diameter of human hair is around $60 \mu \mathrm{m}$ ) of micro- tomed hair from an adult. The sample was embedded in epoxy resin prior to microtoming. The well-known hair morphology is detected, which consists of the so-called medulla in the very interior (not shown in the present image), the cortex (consisting of a microfibrillar structure) and the outer cuticle. Five layers from the cuticle can be resolved in the present image. Small holes within the cortex regime are observed indicating the less dense morphology. The encircled area highlights some pigments embedded in the cuticle regime.
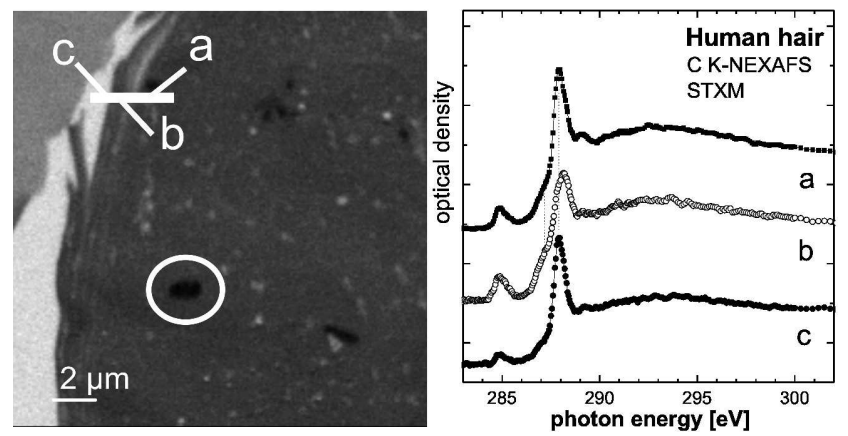

Fig. 5. STXM investigations of human hair. Left: STXM transmission image (scanned area: $20 \times 20 \mu \mathrm{m}^{2}$, photon energy $287.7 \mathrm{eV}$ ); the circle highlights incorporated pigments. Right: C $K$ NEXAFS spectra extracted from a line scan along the cuticle - the respective positions are marked in the image on the left side.

A line scan was performed along the line in the upper left and $\mathrm{C} K$ NEXAFS spectra were recorded from the positions denoted $a, b$, and $c$. The spectra are quite similar and dominated by an intense resonance at about $287.9 \mathrm{eV}$, a weaker shoulder at $287.1 \mathrm{eV}$ and small intensity at $284.85 \mathrm{eV}$. Since it is known that one of the main constituents of human hair is the S-containing amino acid cystine, we can directly compare the obtained spectra with those from NEXAFS databases. Zubavichus et al. [24] have analyzed a large variety of amino acids and, indeed, the obtained spectra from our sample is consistent with the reported NEXAFS from cystine. In Ref. [24] the prominent resonance is assigned to $\sigma_{(\mathrm{C}-\mathrm{S})}^{*}$ excitations. The small intensity around $285 \mathrm{eV}$ might also be due to radiation damage during line scans. From the present data sets we cannot explain the different resonance shape (extra intensity at higher photon energy at the major resonance) at spot $b$. Additional spectroscopic analysis at the $\mathrm{O} K$-edge may give further insight into the local variations and more detailed chemical assignment.

\section{Summary and conclusions}

Zone-plate based scanning transmission X-ray microspectroscopy in the soft $\mathrm{X}$-ray regime is rapidly developing into a powerful complementary technique for nanoanalytical studies in material science. The recently installed PolLux-STXM at the Swiss Light Source will 
increase the availability in Europe. The presented examples on complex micro- and nanostructured materials underline the importance to combine spectral and spatial information to find structure-property relationships. The wide energy range available at PolLux as well as the potential to fast helicity switching will allow improved micromagnetic analysis of ferromagnetic materials to investigate the magnetization dynamics on the subnanosecond timescale. Also more biologically or medical relevant studies may be considered.

\section{Acknowledgments}

The work is funded by the BMBF under projects 05KS4WE1/6 and 05 KS7WE1. We gratefully acknowledge experimental support by B. Graf (Univ. Erlangen), A.L.D. Kilcoyne (ALS, Berkeley), and J. Raabe (SLS/PSI). Sample materials were partly prepared by A. Fery (Univ. Bayreuth), P. Fernandes (MPI, Golm), and G. Paradossi (Univ. Tor Vergata, Roma). Microtoming of the hair sample was performed by Mrs. C. Kunert (Univ. Bayreuth).

\section{References}

[1] J. Stöhr, NEXAFS Spectroscopy, Springer-Verlag, Berlin 1992.

[2] S. Anders, H.A. Padmore, et al., Rev. Sci. Instrum. 70, 3973 (1999).

[3] H. Marchetto, U. Groh, Th. Schmidt, H. Kuhlenbeck, R. Fink, H.-J. Freund, E. Umbach, Chem. Phys. 325, 178 (2006).

[4] J. Kirz, H. Rarback, Rev. Sci. Instrum. 56, 1 (1985).

[5] H. Ade, X. Zhang, S. Cameron, C. Costello, J. Kirz, S. Williams, Science 258, 972 (1992).

[6] A.L.D. Kilcoyne, T. Tyliszczak, W.F. Steele, S. Fakra, P. Hitchcock, K. Franck, E. Anderson, B. Harteneck, J. Synchrotron Radiat. 10, 125 (2003).

[7] T. Beetz, M. Feser, H. Fleckenstein, B. Hornberger, C. Jacobsen, J. Kirz, M. Lerotic, E. Lima, M. Lu, D. Sayre, D. Shapiro, A. Stein, D. Tennant, S. Wirick, Synchrotron Radiat. News 16, 11 (2003).

[8] K.V. Kaznatcheev, Ch. Karunakaran, et al., Nucl. Instrum. Methods Phys. Res. A 582, 96 (2007).
[9] U. Wiesemann, J. Thieme, P. Guttmann, B. Niemann, D. Rudolph, G. Schmahl, AIP Conf. Proc. 507, 430 (2000).

[10] B. Kaulich, D. Bacescu, D. Cocco, J. Susini, M. Salom, O. Dhez, C. David, T. Weitkamp, E. Di Fabrizio, S. Cabrini, G. Morrison, P. Charalambous, J. Thieme, T. Wilhein, J. Kovac, M. Podnar, M. Kiskinova, J. Phys. (France) IV 104, 103 (2003).

[11] J. Raabe, G. Tzvetkov, U. Flechsig, M. Böge, A. Jaggi, B. Sarafimov, C. Quitmann, M. HeubergerVernooij, T. Huthwelker, H. Ade, D. Kilcoyne, T. Tyliszczak, Rev. Sci. Instrum. 79, 113704 (2008).

[12] K. Jefimovs, J. Vila-Comamala, T. Pilvi, J. Raabe, M. Ritala, C. David, Phys. Rev. Lett. 99, 264801 (2007).

[13] H. Ade, A.P. Hitchcock, Polymer 49, 643 (2008).

[14] B. Van Waeyenberge, A. Puzic, H. Stoll, K.W. Chou, T. Tyliszczak, R. Hertel, M. Fähnle, H. Brückl, K. Rott, G. Reiss, I. Neudecker, D. Weiss, C.H. Back, G. Schütz, Nature 444, 461 (2006).

[15] H. Klauk, U. Zschieschang, J. Pflaum, M. Halik, Nature 445, 745 (2007).

[16] http://www.micronal.com.

[17] G. Tzvetkov, B. Graf, R. Wiegner, J. Raabe, C. Quitmann, R. Fink, Micron 39, 275 (2008).

[18] J. Stöhr, D.A. Outka, K. Baberschke, D. Arvanitis, J.A. Horsley, Phys. Rev. B 36, 2976 (1987).

[19] K. Weiss, P.S. Bagus, Ch. Wöll, J. Chem. Phys. 111, 6834 (1999).

[20] S.G. Urquhart, R. Gillies, J. Phys. Chem. A $\mathbf{1 0 9}$ 2151 (2005).

[21] G. Tzvetkov, B. Graf, P. Fernandes, A. Fery, F. Cavalieri, G. Paradossi, R. Fink, Soft Matter 4, 510 (2008).

[22] P. Fernandez, G. Tzvetkov, R.H. Fink, G. Paradossi, A. Fery, Langmuir 24, 13677 (2008).

[23] M.E. Rousseau, D. Hernández Cruz, M.M. West, A.P. Hitchcock, M. Pézolet, J. Am. Chem. Soc. 129 , 3897 (2007)

[24] Y. Zubavichus, A. Shaporenko, M. Grunze, M. Zharnikov, J. Chem. Phys. A (Lett.) $\mathbf{1 0 9}$ 6998 (2005). 